\title{
Energy-momentum tensor on the lattice: non- perturbative renormalization in Yang-Mills theory
}

\author{
Leonardo Giusti ${ }^{a, b}$ and Michele Pepe ${ }^{b}$ \\ a Dipartimento di Fisica, Università di Milano-Bicocca, \\ Piazza della Scienza 3, I-20126 Milano, Italy \\ ${ }^{b}$ INFN, Sezione di Milano-Bicocca, \\ Piazza della Scienza 3, I-20126 Milano, Italy
}

\begin{abstract}
We construct an energy-momentum tensor on the lattice which satisfies the appropriate Ward Identities (WIs) and has the right trace anomaly in the continuum limit. It is defined by imposing suitable WIs associated to the Poincare invariance of the continuum theory. These relations come forth when the length of the box in the temporal direction is finite, and they take a particularly simple form if the coordinate and the periodicity axes are not aligned. We implement the method for the SU(3) Yang-Mills theory discretized with the standard Wilson action in presence of shifted boundary conditions in the (short) temporal direction. By carrying out extensive numerical simulations, the renormalization constants of the traceless components of the tensor are determined with a precision of roughly half a percent for values of the bare coupling constant in the range $0 \leq g_{0}^{2} \leq 1$.
\end{abstract}

\section{INTRODUCTION}

On the lattice the Poincaré group is explicitly broken into discrete subgroups, and the full symmetry is recovered only in the continuum limit. As a consequence, a given definition of the energy-momentum tensor needs to be properly renormalized to guarantee that the associated charges are the generators of the Poincaré group in the continuum limit, and that the trace anomaly is correctly reproduced.

In order to construct the renormalized energymomentum tensor, the way to proceed is to impose suitable WIs at fixed lattice spacing that hold up to cutoff effects which vanish in the continuum limit [1]. This program can be realized in practice if the WIs involve correlators which in turn are simple enough to be computed numerically with good precision.

When the theory is considered in a finite box, the Euclidean Lorentz symmetry is also softly broken by its shape. If the length in one (temporal) direction $L_{0}$ is chosen to be shorter than the typical scale of the theory (thermal theory), interesting WIs follows 2-4]. They become particularly simple and of practical use if the periodicity axes are tilted with respect to the lattice grid, i.e. if the hard breaking of the Poincaré symmetry due to the lattice discretization and the soft one due to the finite temporal direction are not aligned. This set-up has a natural implementation in the Euclidean path-integral formulation in terms of shifted boundary conditions [2, 5].

Here we define the renormalized energy-momentum tensor of the Yang-Mills theory non-perturbatively by working in this framework. This is achieved by supplementing the set of WIs found in Refs [3, 4] with a new one which guarantees that the correct trace anomaly is reproduced in the continuum limit.

We implement this strategy for the SU(3) Yang-Mills theory regularized with the standard Wilson action. We carry out extensive numerical simulations, and compute with high precision the renormalization constants of the traceless components of the tensor. The numerical determination of the renormalization constant of the trace part requires additional simulations, and it is left for a forthcoming publication. Over the last year an alternative method, based on the Yang-Mills gradient flow, has also been proposed for renormalizing non-perturbatively the energy-momentum tensor [ $[6]$ [ $]$.

\section{WARD IDENTITIES IN PRESENCE OF A NON-ZERO SHIFT}

In this section we consider the SU(3) Yang-Mills theory in the continuum. The definitions of the action and of the partition function $Z$ are reported in Appendices A and $B$ together with other conventions. Here we are interested in the thermal theory defined in the path integral formalism with shifted boundary conditions

$$
A_{\mu}\left(L_{0}, \boldsymbol{x}\right)=A_{\mu}\left(0, \boldsymbol{x}-L_{0} \boldsymbol{\xi}\right)
$$

along the compact (temporal) direction of length $L_{0}$ with shift $\boldsymbol{\xi} \in \mathbb{R}^{3}$. The free-energy density is given by

$$
f\left(L_{0}, \boldsymbol{\xi}\right)=-\frac{1}{L_{0} V} \ln Z\left(L_{0}, \boldsymbol{\xi}\right),
$$

where $V$ is the spatial volume of the box. In the thermodynamic limit, which is always assumed in this section, the invariance of the dynamics under the $\mathrm{SO}(4)$ group implies [4]

$$
f\left(L_{0}, \boldsymbol{\xi}\right)=f\left(L_{0} \sqrt{1+\boldsymbol{\xi}^{2}}, \mathbf{0}\right) .
$$


When $\boldsymbol{\xi} \neq 0$, odd derivatives in $\xi_{k}$ do not vanish, and the following interesting relations hold $[2-4]\left(x_{0} \neq 0\right)$ :

$$
\begin{aligned}
L_{0}\left\langle T_{0 k}\right\rangle_{\boldsymbol{\xi}} & =\frac{1}{V} \frac{\partial}{\partial \xi_{k}} \ln Z\left(L_{0}, \boldsymbol{\xi}\right), \\
\frac{\partial}{\partial \xi_{k}}\langle O\rangle_{\boldsymbol{\xi}} & =L_{0}\left\langle\bar{T}_{0 k}\left(x_{0}\right) O(0)\right\rangle_{\boldsymbol{\xi}, c},
\end{aligned}
$$

where $T_{\mu \nu}$ is the energy-momentum tensor, $\bar{T}_{\mu \nu}=$ $\int d^{3} x T_{\mu \nu}(x), O$ is a generic gauge invariant operator, and the subscript $c$ indicates a connected correlation function. By deriving once with respect to $L_{0}$ and to $\xi_{k}$ one obtains the relation (no summation over repeated $k$ here) [4]

$$
\left\langle T_{0 k}\right\rangle_{\boldsymbol{\xi}}=\frac{\xi_{k}}{1-\xi_{k}^{2}}\left\{\left\langle T_{00}\right\rangle_{\boldsymbol{\xi}}-\left\langle T_{k k}\right\rangle_{\boldsymbol{\xi}}\right\} .
$$

In the equations above and in the rest of this paper we focus on correlation functions of the energy-momentum tensor $T_{\mu \nu}$ with gauge-invariant operators inserted at a physical distance. As reviewed in Appendix C, it is appropriate in those cases to consider the symmetric gaugeinvariant definition of the energy-momentum tensor given by

$$
T_{\mu \nu}=\frac{1}{g_{0}^{2}}\left\{F_{\mu \alpha}^{a} F_{\nu \alpha}^{a}-\frac{1}{4} \delta_{\mu \nu} F_{\alpha \beta}^{a} F_{\alpha \beta}^{a}\right\} .
$$

By deriving two times with respect to the shift components and by using Eq. (4), one obtains [4]

$$
\left\langle T_{0 k}\right\rangle_{\boldsymbol{\xi}}=\frac{L_{0} \xi_{k}}{2} \sum_{i j}\left\langle\bar{T}_{0 i} T_{0 j}\right\rangle_{\boldsymbol{\xi}, c}\left[\delta_{i j}-\frac{\xi_{i} \xi_{j}}{\boldsymbol{\xi}^{2}}\right]
$$

where on the r.h.s. the two fields are inserted at different times. Analogously one shows that $\left(x_{0} \neq 0\right)$

$$
\begin{array}{r}
L_{0}\left\langle\bar{T}_{0 k}\left(x_{0}\right) T_{\mu \mu}(0)\right\rangle_{\boldsymbol{\xi}, c}=\left\{6-\frac{1+\xi^{2}}{\xi_{k}^{2}}\right\}\left\langle T_{0 k}\right\rangle_{\boldsymbol{\xi}}+ \\
L_{0} \frac{1+\xi^{2}}{\xi_{k}}\left\langle\bar{T}_{0 k}\left(x_{0}\right) T_{0 k}(0)\right\rangle_{\boldsymbol{\xi}, c},
\end{array}
$$

which can also be put in the more suggestive form

$$
\frac{\partial}{\partial \xi_{k}}\left\langle T_{\mu \mu}\right\rangle_{\boldsymbol{\xi}}=\frac{1}{\left(1+\xi^{2}\right)^{2}} \frac{\partial}{\partial \xi_{k}}\left[\frac{\left(1+\xi^{2}\right)^{3}}{\xi_{k}}\left\langle T_{0 k}\right\rangle_{\boldsymbol{\xi}}\right]
$$

\section{A. Finiteness of $T_{\mu \nu}$ and trace anomaly}

In dimensional regularization, the energy-momentum tensor in Eq. (6) is decomposed as

$$
T_{\mu \nu}=\tau_{\mu \nu}+\delta_{\mu \nu} \tau
$$

where

$$
\begin{aligned}
\tau_{\mu \nu} & =\frac{1}{g_{0}^{2}}\left\{F_{\mu \alpha}^{a} F_{\nu \alpha}^{a}-\frac{1}{D} \delta_{\mu \nu} F_{\alpha \beta}^{a} F_{\alpha \beta}^{a}\right\}, \\
\tau & =\frac{\epsilon}{2 D g_{0}^{2}} F_{\alpha \beta}^{a} F_{\alpha \beta}^{a}
\end{aligned}
$$

are two fields transforming as a two-index symmetric and a singlet irreducible representation of the $\mathrm{SO}(\mathrm{D})$ group respectively, and $D=4-2 \epsilon$.

The field $\tau_{\mu \nu}$ is a dimension-four gauge invariant operator which is multiplicatively renormalizable. The WI in Eq. (7) fixes its renormalization constant to 1. This in turn implies that $g_{0}^{2} \tau_{\mu \nu}$ renormalizes as

$$
\begin{aligned}
\left\{F_{\mu \alpha}^{a} F_{\nu \alpha}^{a}-\frac{1}{D} \delta_{\mu \nu} F_{\alpha \beta}^{a} F_{\alpha \beta}^{a}\right\}^{\mathrm{R}}= & Z_{g}\left\{F_{\mu \alpha}^{a} F_{\nu \alpha}^{a}\right. \\
& \left.-\frac{1}{D} \delta_{\mu \nu} F_{\alpha \beta}^{a} F_{\alpha \beta}^{a}\right\},
\end{aligned}
$$

where $Z_{g}$ is the renormalization constant of the coupling, see Appendix D. By defining the renormalization group invariant (RGI) operator as [9]

$$
\begin{aligned}
\left\{F_{\mu \alpha}^{a} F_{\nu \alpha}^{a}-\frac{1}{D} \delta_{\mu \nu} F_{\alpha \beta}^{a} F_{\alpha \beta}^{a}\right\}^{\mathrm{RGI}}= & \frac{1}{2 b_{0} g^{2}}\left\{F_{\mu \alpha}^{a} F_{\nu \alpha}^{a}\right. \\
& \left.-\frac{1}{D} \delta_{\mu \nu} F_{\alpha \beta}^{a} F_{\alpha \beta}^{a}\right\}^{\mathrm{R}},
\end{aligned}
$$

we finally arrive to

$$
\tau_{\mu \nu}=\tau_{\mu \nu}^{\mathrm{R}}=2 b_{0}\left\{F_{\mu \alpha}^{a} F_{\nu \alpha}^{a}-\frac{1}{D} \delta_{\mu \nu} F_{\alpha \beta}^{a} F_{\alpha \beta}^{a}\right\}^{\mathrm{RGI}},
$$

where $b_{0}$ is the first coefficient of the $\beta$-function given in Eq. (D4). The field $\tau$ is also dimension-four and gauge invariant, but it is a singlet under $\mathrm{SO}(\mathrm{D})$. Therefore it mixes with itself and with the identity operator. The Eq. (8) fixes the multiplicative renormalization constant to 1 , while a natural prescription for the identity subtraction is

$$
\tau^{\mathrm{R}}=\tau-\langle\tau\rangle_{0}
$$

where $\langle\ldots\rangle_{0}$ indicates the vacuum expectation value for $L_{0} \rightarrow \infty$ (zero temperature). This in turn implies that one can define

$$
\left\{F_{\alpha \beta}^{a} F_{\alpha \beta}^{a}\right\}^{\mathrm{R}}=Z_{E}^{-1}\left\{F_{\alpha \beta}^{a} F_{\alpha \beta}^{a}-\left\langle F_{\alpha \beta}^{a} F_{\alpha \beta}^{a}\right\rangle_{0}\right\},
$$

and Eq. (15) becomes

$$
\tau^{\mathrm{R}}=\frac{\epsilon Z_{g} Z_{E}}{2 D \mu^{2 \epsilon} g^{2}}\left\{F_{\alpha \beta}^{a} F_{\alpha \beta}^{a}\right\}^{\mathrm{R}} .
$$

By using the result in Eq. (D12) of Appendix D. one obtains the well known result for the trace anomaly [10, 11 .

$$
T_{\mu \mu}=4 \tau^{\mathrm{R}}=-\frac{\beta}{2 g^{3}}\left\{F_{\alpha \beta}^{a} F_{\alpha \beta}^{a}\right\}^{\mathrm{R}} .
$$

By defining the renormalization group invariant operator as [9]

$$
\left\{F_{\alpha \beta}^{a} F_{\alpha \beta}^{a}\right\}^{\mathrm{RGI}}=-\frac{\beta}{b_{0} g^{3}}\left\{F_{\alpha \beta}^{a} F_{\alpha \beta}^{a}\right\}^{R},
$$


we can finally write

$$
T_{\mu \mu}=4 \tau^{\mathrm{R}}=\frac{b_{0}}{2}\left\{F_{\alpha \beta}^{a} F_{\alpha \beta}^{a}\right\}^{\mathrm{RGI}} .
$$

The WIs in Eqs. (7) and (8) fix unambiguously the renormalization constants of the composite fields entering the energy-momentum tensor definition so that the correct trace anomaly is reproduced. The Eqs. (18)-(20) hold to all orders in perturbation theory.

\section{THE ENERGY MOMENTUM TENSOR ON THE LATTICE}

We regularize the $\mathrm{SU}(3)$ Yang-Mills theory on a finite four-dimensional lattice of spatial volume $V=L^{3}$, temporal direction $L_{0}$, and spacing $a$. The gauge field satisfies periodic boundary conditions in the three spatial directions and shifted boundary conditions in the compact direction

$$
U_{\mu}\left(L_{0}, \boldsymbol{x}\right)=U_{\mu}\left(0, \boldsymbol{x}-L_{0} \boldsymbol{\xi}\right),
$$

where $U_{\mu}\left(x_{0}, \boldsymbol{x}\right)$ are the link variables. The action is discretized through the standard Wilson plaquette

$$
S[U]=\frac{\beta}{2} a^{4} \sum_{x} \sum_{\mu, \nu}\left[1-\frac{1}{3} \operatorname{Re} \operatorname{Tr}\left\{U_{\mu \nu}(x)\right\}\right],
$$

where the trace is over the color index, and $\beta=6 / g_{0}^{2}$ with $g_{0}$ being the bare coupling constant. The plaquette is defined as a function of the gauge links, and it given by

$$
U_{\mu \nu}(x)=U_{\mu}(x) U_{\nu}(x+a \hat{\mu}) U_{\mu}^{\dagger}(x+a \hat{\nu}) U_{\nu}^{\dagger}(x),
$$

where $\mu, \nu=0, \ldots, 3, \hat{\mu}$ is the unit vector along the direction $\mu$, and $x$ is the space-time coordinate. The gluon field strength tensor is defined a: 1$]$ [1]

$$
F_{\mu \nu}^{a}(x)=-\frac{i}{4 a^{2}} \operatorname{Tr}\left\{\left[Q_{\mu \nu}(x)-Q_{\nu \mu}(x)\right] T^{a}\right\}
$$

where

$$
\begin{aligned}
& Q_{\mu \nu}(x)=U_{\mu}(x) U_{\nu}(x+a \hat{\mu}) U_{\mu}^{\dagger}(x+a \hat{\nu}) U_{\nu}^{\dagger}(x) \\
& +U_{\nu}(x) U_{\mu}^{\dagger}(x-a \hat{\mu}+a \hat{\nu}) U_{\nu}^{\dagger}(x-a \hat{\mu}) U_{\mu}(x-a \hat{\mu}) \\
& +U_{\mu}^{\dagger}(x-a \hat{\mu}) U_{\nu}^{\dagger}(x-a \hat{\mu}-a \hat{\nu}) U_{\mu}(x-a \hat{\mu}-a \hat{\nu}) U_{\nu}(x-a \hat{\nu}) \\
& +U_{\nu}^{\dagger}(x-a \hat{\nu}) U_{\mu}(x-a \hat{\nu}) U_{\nu}(x+a \hat{\mu}-a \hat{\nu}) U_{\mu}^{\dagger}(x) .
\end{aligned}
$$

\footnotetext{
${ }^{1}$ We use the same notation for lattice and continuum quantities, since any ambiguity is resolved from the context. As usual, the continuum limit value of a renormalized lattice quantity, identified with the subscript $\mathrm{R}$, is the one to be identified with its continuum counterpart.
}

The target energy-momentum tensor in the continuum is a gauge-invariant operator of dimension 4, which is a combination of a traceless two-index symmetric and a singlet irreducible representation of $\mathrm{SO}(4)$. When $\mathrm{SO}(4)$ is broken to the hypercubic group $\mathrm{SW}_{4}$, the traceless two-index symmetric representation splits into a sextet (non-diagonal components) and a triplet (diagonal traceless components). At finite lattice spacing, the energy-momentum tensor is thus a combination of gaugeinvariant operators of dimension $d \leq 4$ which, under the hypercubic group, transform as one of those two representations and the singlet. In the $\mathrm{SU}(3)$ Yang-Mills theory there are only three such operators (no summation over repeated $\mu$ and $\nu$ here) [1, 12]:

$$
\begin{aligned}
T_{\mu \nu}^{[1]} & =\left(1-\delta_{\mu \nu}\right) \frac{1}{g_{0}^{2}}\left\{F_{\mu \alpha}^{a} F_{\nu \alpha}^{a}\right\} \\
T_{\mu \nu}^{[2]} & =\delta_{\mu \nu} \frac{1}{4 g_{0}^{2}} F_{\alpha \beta}^{a} F_{\alpha \beta}^{a} \\
T_{\mu \nu}^{[3]} & =\delta_{\mu \nu} \frac{1}{g_{0}^{2}}\left\{F_{\mu \alpha}^{a} F_{\mu \alpha}^{a}-\frac{1}{4} F_{\alpha \beta}^{a} F_{\alpha \beta}^{a}\right\}
\end{aligned}
$$

and the identity. The sextet $T_{\mu \nu}^{[1]}$ and the triplet $T_{\mu \nu}^{[3]}$ renormalize multiplicatively, while the singlet $T_{\mu \nu}^{[2]}$ mixes also with the identity. The renormalized energymomentum tensor can finally be written as

$$
T_{\mu \nu}^{\mathrm{R}}=Z_{T}\left\{T_{\mu \nu}^{[1]}+z_{T} T_{\mu \nu}^{[3]}+z_{S}\left[T_{\mu \nu}^{[2]}-\left\langle T_{\mu \nu}^{[2]}\right\rangle_{0}\right]\right\} .
$$

The renormalization constants $Z_{T}, z_{T}$ and $z_{S}$ are finite and depend on $g_{0}^{2}$ only. At one loop in perturbation theory their expressions are [1, 12]

$$
\begin{aligned}
& Z_{T}\left(g_{0}^{2}\right)=1+0.27076 g_{0}^{2}, \\
& z_{T}\left(g_{0}^{2}\right)=1-0.03008 g_{0}^{2}, \\
& z_{S}\left(g_{0}^{2}\right)=\frac{b_{0}}{2} g_{0}^{2} .
\end{aligned}
$$

\section{A. Non-perturbative renormalization conditions}

The renormalization constants $Z_{T}, z_{T}$ and $z_{S}$ can be determined non-perturbatively by requiring that on the lattice the WIs in Eqs. (4), (5), and (9) hold up to discretization effects which vanish in the continuum limit. The renormalization constant of the sextet is fixed to be [13] (see also [14])

$$
Z_{T}\left(g_{0}^{2}\right)=-\frac{\Delta f}{\Delta \xi_{k}} \frac{1}{\left\langle T_{0 k}^{[1]}\right\rangle_{\xi}},
$$

where the derivative in the shift in Eq. (4) is discretized by the symmetric finite difference

$$
\frac{\Delta f}{\Delta \xi_{k}}=\frac{1}{2 a V} \ln \left[\frac{Z\left(L_{0}, \boldsymbol{\xi}-a \hat{k} / L_{0}\right)}{Z\left(L_{0}, \boldsymbol{\xi}+a \hat{k} / L_{0}\right)}\right]
$$



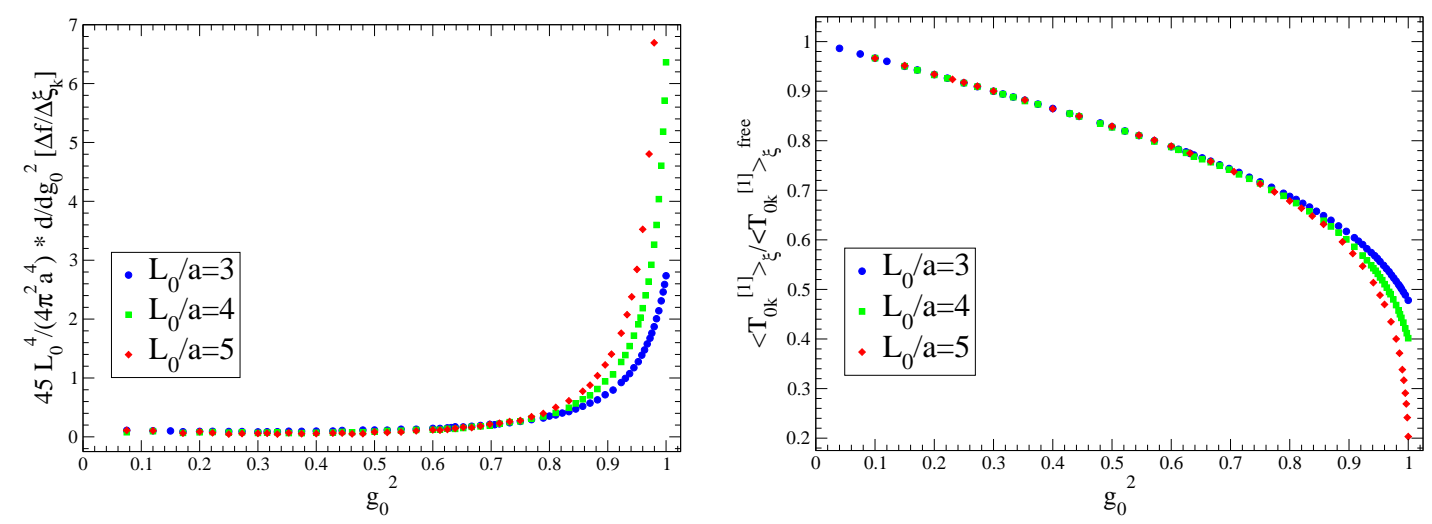

FIG. 1. Left: the derivative of $\Delta f / \Delta \xi_{k}$ (normalized to its Stefan-Boltzmann value) with respect to $g_{0}^{2}$ as a function of the bare coupling. Right: $\left\langle T_{0 k}^{[1]}\right\rangle_{\boldsymbol{\xi}}$ normalized to its tree-level expression as a function of $g_{0}^{2}$. The data are generated on lattices with $L_{0} / a=3$ (blue), 4 (green) and 5 (red), $L / a=48$, and $\boldsymbol{\xi}=(1,0,0)$. Statistical errors are smaller than symbols.

to ensure that discretization effects start at $O\left(a^{2}\right)$. In the thermodynamic limit, which is always assumed in this section, the triplet is renormalized by requiring that Eq. (5) holds up to harmless discretization effects, i.e.

$$
z_{T}\left(g_{0}^{2}\right)=\frac{1-\xi_{k}^{2}}{\xi_{k}} \frac{\left\langle T_{0 k}^{[1]}\right\rangle_{\boldsymbol{\xi}}}{\left\langle T_{00}^{[3]}\right\rangle_{\boldsymbol{\xi}}-\left\langle T_{k k}^{[3]}\right\rangle_{\boldsymbol{\xi}}} .
$$

$$
z_{S}=\frac{1}{\left(1+\xi^{2}\right)^{2}} \frac{\left[\frac{\left(1+\xi^{\prime 2}\right)^{3}}{\xi_{k}^{\prime}}\left\langle T_{0 k}^{[1]}\right\rangle_{\boldsymbol{\xi}^{\prime}}\right]_{\boldsymbol{\xi}^{\prime}=\boldsymbol{\xi}+a \hat{k} / L_{0}}-\left[\frac{\left(1+\xi^{\prime 2}\right)^{3}}{\xi_{k}^{\prime}}\left\langle T_{0 k}^{[1]}\right\rangle_{\boldsymbol{\xi}^{\prime}}\right]_{\boldsymbol{\xi}^{\prime}=\boldsymbol{\xi}-a \hat{k} / L_{0}}}{\left\langle T_{\mu \mu}^{[2]}\right\rangle_{\boldsymbol{\xi}+a \hat{k} / L_{0}}-\left\langle T_{\mu \mu}^{[2]}\right\rangle_{\boldsymbol{\xi}-a \hat{k} / L_{0}}}
$$

At finite $L_{0}$, the renormalization constants depend on the bare coupling constant and on $\left(a / L_{0}\right)^{2}$ due to discretization effects. Our prescription is to define them in the limit2 $L_{0} \rightarrow \infty$ at fixed $g_{0}^{2}$.

\section{NUMERICAL COMPUTATION}

In this section we describe how the strategy outlined above has been implemented in practice to determine the renormalization constants $Z_{T}$ and $z_{T}$. In all simulations the basic Monte Carlo step is a combination of heatbath and over-relaxation updates of the link variables using the Cabibbo-Marinari scheme [15]. A single sweep is made of 1 heatbath and 3 over-relaxation updates of all link variables. All lattices have an inverse temporal length $1 / L_{0}>T_{c}$, where $T_{c}$ is the critical temperature of the theory. We have checked explicitly

\footnotetext{
${ }^{2}$ Notice that in Ref. [13] a different condition was imposed. Since we were interested in $Z_{T}\left(g_{0}^{2}\right)$ in a limited range of $g_{0}^{2}$, we defined $Z_{T}\left(g_{0}^{2}\right)$ as in Eq. (29) but at finite $L_{0}$.
}

By choosing one possibility of discretizing Eq. (9), the singlet renormalization constant is fixed to be the autocorrelation times of the primary observables by profiting from the long Monte Carlo histories, which are typically made of $O\left(10^{5}\right)$ sweeps. No long autocorrelations were observed 3 . For the statistical analysis we have blocked together the primary observables generated in several hundreds of consecutive sweeps, a value which is always much larger than the autocorrelation times measured. It is important to notice that the determinations of $Z_{T}$ and $z_{T}$ require (see below) the computation of expectation values of single local operators only. Indeed increasing the spatial size of the lattice does not increase the computational effort at fixed statistical accuracy.

\section{A. Determination of $Z_{T}$}

The direct determination of $\Delta f / \Delta \xi_{k}$ in Eq. (29) would involve the computation of the ratio of two partition functions with different shifts at the same value of $L_{0} / a$ and

\footnotetext{
${ }^{3}$ At these values of $L_{0}$ fluctuations of the topological charge away from zero are heavily suppressed.
} 

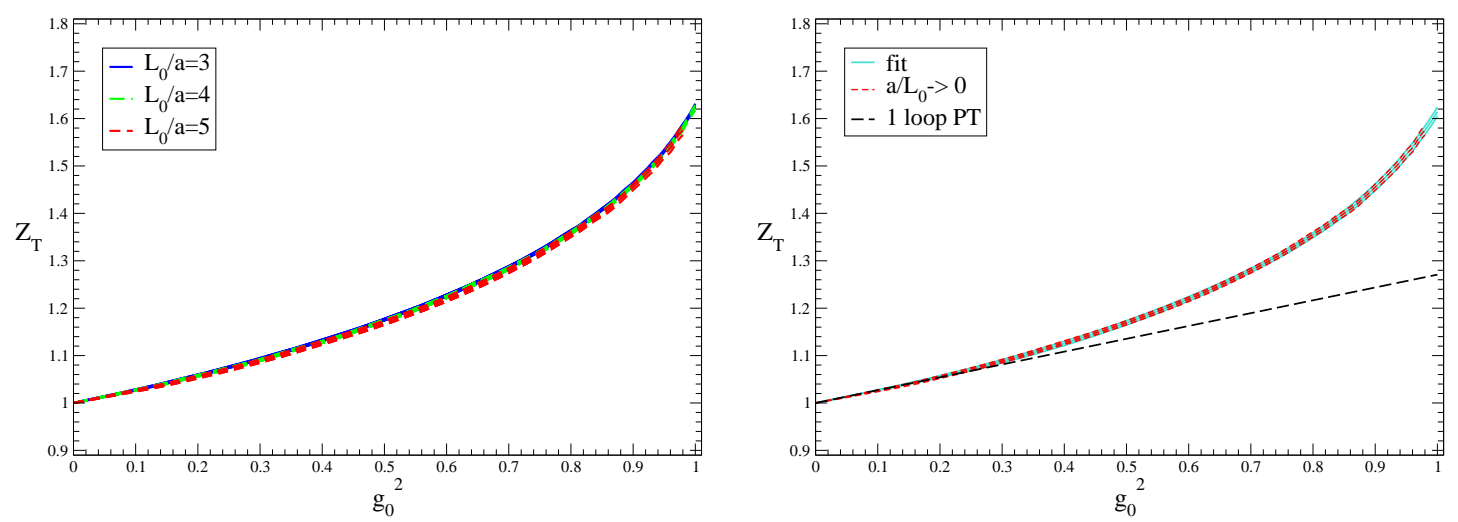

FIG. 2. Left: the renormalization factor $Z_{T}\left(g_{0}^{2}\right)$ as a function of the bare coupling $g_{0}^{2}$ for $L_{0} / a=3$ (blue), 4 (green) and 5 (red). Right: the renormalization factor $Z_{T}\left(g_{0}^{2}\right)$ defined in the limit $a / L_{0} \rightarrow 0$ together with the fit to the formula in Eq. (38) and the one-loop analytic result in Eq. (28).

$g_{0}^{2}$. Since the relevant phase spaces in the path integral of the two systems overlap very poorly, the ratio cannot be estimated in a single Monte Carlo simulation. A possible way out is to define a series of physical systems with actions which interpolate between the two original ones, and then use the Monte Carlo procedure of Refs. [2, 16, 17]. The calculation, however, becomes quickly demanding for large lattices since the numerical cost increases quadratically with the spatial volume.

To bypass this problem we can profit from the fact that $\Delta f / \Delta \xi_{k}$ is a smooth function of $g_{0}^{2}$ at fixed values of $L_{0} / a$ and $L / a$ in the range of chosen values. Its derivative with respect to $g_{0}^{2}$ can be written as

$$
\frac{d}{d g_{0}^{2}} \frac{\Delta f}{\Delta \xi_{k}}=\frac{1}{2 a L^{3} g_{0}^{2}}\left\{\langle S\rangle_{\boldsymbol{\xi}-a / L_{0} \hat{k}}-\langle S\rangle_{\boldsymbol{\xi}+a / L_{0} \hat{k}}\right\},
$$

where $\langle S\rangle_{\boldsymbol{\xi}}$ stands for the expectation value of the action in Eq. (22). Although the quantities on the r.h.s. of Eq. (33) have values which are close to each other, their difference can be computed at a few permille accuracy with a moderate numerical effort. The difference $\left\{\langle S\rangle_{\boldsymbol{\xi}-a / L_{0} \hat{k}}-\langle S\rangle_{\boldsymbol{\xi}+a / L_{0} \hat{k}}\right\}$ has been computed for $\boldsymbol{\xi}=(1,0,0)$ and $L / a=48$ at 63,59 and 48 values of $g_{0}^{2}$ for $L_{0} / a=3,4$ and 5 respectively. A sample of values is reported in Table [1] while all of them are shown in the left plot of Fig. 1 At each value of $L_{0} / a$ the points are interpolated with a cubic spline, and the resulting curve is integrated over $g_{0}^{2}$. The free-case value is computed analytically by using Eq. (E12), and is added to the integral. The systematics induced by the interpolation and the numerical integration of the data is negligible with respect to the statistical error.

To complete the calculation of $Z_{T}\left(g_{0}^{2}\right)$, the expectation value $\left\langle T_{0 k}^{[1]}\right\rangle_{\xi}$ is measured in a dedicated set of simulations. It is computed for $\boldsymbol{\xi}=(1,0,0)$ and $L / a=48$ at 66,60 and 38 values of $g_{0}^{2}$ for $L_{0} / a=3,4$ and 5 respectively. A sample of values is reported in Table II and all of them are shown in the right plot of Fig. 1. By interpolating the results with a cubic spline, the renor- malization constant $Z_{T}\left(g_{0}^{2}\right)$ is finally determined by the tree-level improved version of Eq. (29) given by

$$
Z_{T}\left(g_{0}^{2}\right)=-\left\{\frac{\Delta f}{\Delta \xi_{k}} \frac{1}{\left\langle T_{0 k}^{[1]}\right\rangle_{\boldsymbol{\xi}}}-\text { free case }\right\} .
$$

The result:4 for $Z_{T}\left(g_{0}^{2}\right)$ at $L / a=48$ and $L_{0} / a=3,4$ and 5 are shown in the left plot of Fig. 2. At the larger value of $L_{0} / a=5$, discretization effects in $a / L_{0}$ are within our statistical errors. Those due to the finiteness of $a / L$ have been checked by computing $Z_{T}$ at $L / a=16$ and $L_{0} / a=3$ in the full range of $g_{0}^{2}$, and at $L_{0} / a=5$ and 6 for $g_{0}^{2}>0.85$. The results at $L_{0} / a=3$ for $L / a=16$ and 48 are statistically compatible, and their central values differ at most by $0.5 \%$ toward the larger values of $g_{0}^{2}$. Since on the lattices with $L_{0} / a=5$ and $L / a=48$ those effects are expected to be suppressed at least by an additional factor of $1 / 8$, we conclude that they are well within the statistical errors. We thus quote the values of $Z_{T}\left(g_{0}^{2}\right)$ at $L_{0} / a=5$ and $L / a=48$ as our best results in the limit $a / L_{0} \rightarrow 0$, see right plot of Fig. 2, Even if defined by renormalization conditions which differ from ours by discretization effects, our values of $Z_{T}\left(g_{0}^{2}\right)$ at $g_{0}^{2}>0.8$ agree with those in Refs. 13, 14] which, however, in many cases have a much larger statistical error.

\section{B. Determination of $z_{T}$}

The renormalization constant $z_{T}$ is computed by imposing the tree-level improved version of Eq. (31) given

\footnotetext{
4 Preliminary results have been reported in Ref. [18]

${ }^{5}$ At this small volume we have computed $\Delta f / \Delta \xi_{k}$ either with the method described in this section, or with the Monte Carlo procedure in Ref. [2]. The numerical results are in agreement within statistical errors.
} 

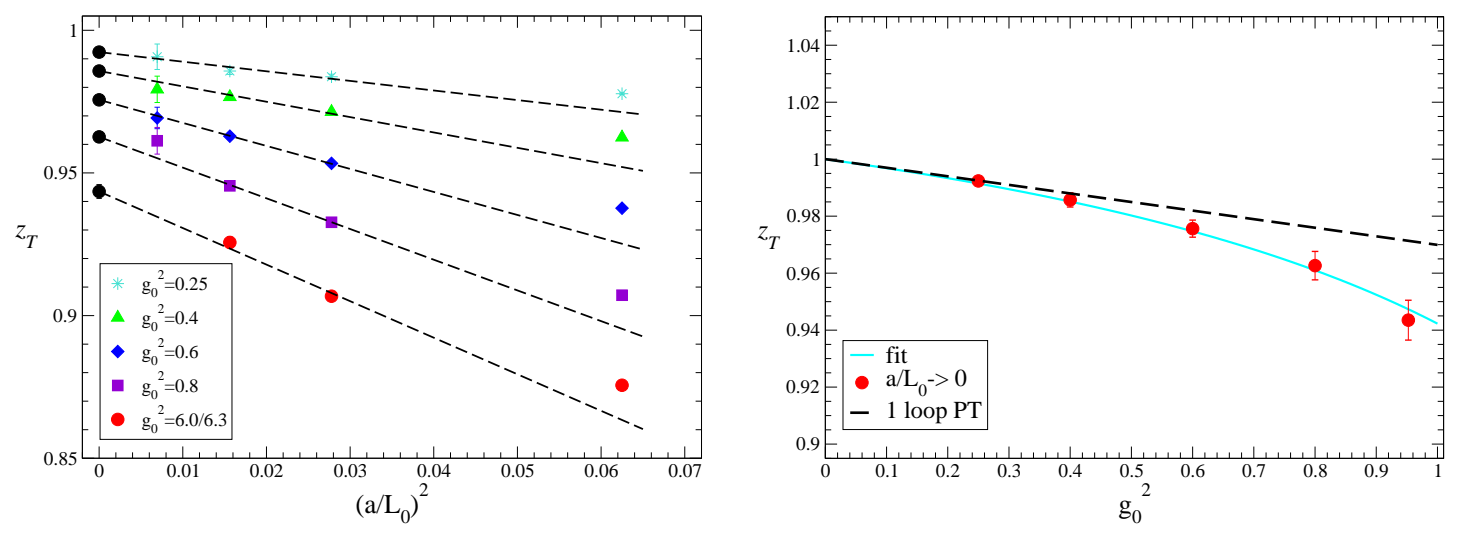

FIG. 3. Left: the renormalization factor $z_{T}\left(g_{0}^{2}, a / L_{0}\right)$ as a function of $\left(a / L_{0}\right)^{2}$ for the five values of $g_{0}^{2}$ indicated in the legend; it is also shown the extrapolation to $\left(a / L_{0}\right)=0$ with the fit function in Eq. (37). Right: the renormalization factor $z_{T}\left(g_{0}^{2}\right)$ at $a / L_{0}=0$ for the 5 values of $g_{0}^{2}$ simulated, together with their fit to the formula in Eq. (39) and with the one-loop analytic result in Eq. (28).

by

$$
z_{T}\left(g_{0}^{2}\right)=\frac{1-\xi_{k}^{2}}{\xi_{k}}\left\{\frac{\left\langle T_{0 k}^{[1]}\right\rangle_{\boldsymbol{\xi}}}{\left\langle T_{00}^{[3]}\right\rangle_{\boldsymbol{\xi}}-\left\langle T_{k k}^{[3]}\right\rangle_{\boldsymbol{\xi}}}-\text { free case }\right\},
$$

with

$$
\frac{L \xi_{k}}{L_{0}\left(1+\xi_{k}^{2}\right)}=q \in \mathbb{Z}
$$

The latter condition guarantees that the WI remains valid at finite volume as it stands [4]. The expectation values of $\left\langle T_{0 k}^{[1]}\right\rangle_{\boldsymbol{\xi}}$ and of the difference $\left\langle T_{00}^{[3]}\right\rangle_{\boldsymbol{\xi}}-\left\langle T_{k k}^{[3]}\right\rangle_{\boldsymbol{\xi}}$ are measured straightforwardly in the same Monte Carlo simulation 6 . The free case is subtracted analytically by using its expression in Appendix E. In practice we chose $\boldsymbol{\xi}=(1 / 2,0,0)$ and $q=8$ so that the ratio of the spatial linear size over the temporal one is fixed to be $L / L_{0}=20$. We simulated 5 values of $g_{0}^{2}$ in the range $0 \leq g_{0}^{2} \leq 1$ with temporal length $L_{0} / a=4,6,8$ and 12 . The results for $z_{T}\left(g_{0}^{2}\right)$ are given in Table III, and they are shown in the left plot of Fig. 3. Discretization effects turn out to be quite larger than for $Z_{T}\left(g_{0}^{2}\right)$ at the smaller values of $L_{0} / a$. Our best extrapolation to $a / L_{0}=0$ is given by the overall fit of the data at $L_{0} / a=6,8$ and 12 to the function

$$
z_{T}\left(g_{0}^{2}, a / L_{0}\right)=z_{T}\left(g_{0}^{2}\right)+b_{1} g_{0}^{2}\left(\frac{a}{L_{0}}\right)^{2} .
$$

The quality of the fit is very good, and it leads to the values of $z_{T}\left(g_{0}^{2}\right)$ shown by the black points in the same plot. To check for the systematics associated to the extrapolation, we have performed a variety of different fits: we

\footnotetext{
${ }^{6}$ It is interesting to notice that the difference $\left\langle T_{00}^{[3]}\right\rangle_{\boldsymbol{\xi}}-\left\langle T_{k k}^{[3]}\right\rangle_{\boldsymbol{\xi}}$ requires roughly 10 times the statistics needed for $\left\langle T_{0 k}^{[1]}\right\rangle_{\boldsymbol{\xi}}$ to meet the same relative statistical error.
}

have removed the points at $L_{0} / a=6$ from our best fit, we have fit each set of points independently with a quadratic function in $\left(a / L_{0}\right)^{2}$, we have amended the combined fit function by adding a quadratic term in $g_{0}^{2}$ to the coefficient of $\left(a / L_{0}\right)^{2}$, and we have added a quadratic term in $\left(a / L_{0}\right)^{2}$ in Eq. (37). The results of all these fits are statistically compatible with those obtained in our best fit to the function in Eq. (37) and the selection of data points chosen. We take the maximum spread of the central values from the various fits as a systematic error due to the extrapolation, and we add it in quadrature to the statistical one. The final results are shown in the right plot of Fig. 3 .

\section{RESULTS AND CONCLUSIONS}

The final results for $Z_{T}\left(g_{0}^{2}\right)$ are shown in the right plot of Fig. 2. They are very well represented by the expression

$$
\begin{aligned}
Z_{T}\left(g_{0}^{2}\right) & =\frac{1-0.4457 g_{0}^{2}}{1-0.7165 g_{0}^{2}}-0.2543 g_{0}^{4} \\
& +0.4357 g_{0}^{6}-0.5221 g_{0}^{8}
\end{aligned}
$$

in the full range $0 \leq g_{0}^{2} \leq 1$, a function which coincide with the expansion in Eq. (28) to order $g_{0}^{2}$. The deviation of the curve from the data is smaller than the statistical accuracy, see right plot of Fig. 2. The error to be attached to $Z_{T}\left(g_{0}^{2}\right)$ computed as in Eq. (38) is $0.4 \%$ up to $g_{0}^{2} \leq 0.85$, while it grows linearly from $0.4 \%$ to $0.7 \%$ in the range $0.85 \leq g_{0}^{2} \leq 1$. Within our statistical errors, the non-perturbative determination starts to deviate significantly from the one-loop result at $g_{0}^{2} \sim 0.25$.

Our best results for $z_{T}\left(g_{0}^{2}\right)$ are shown in the right plot of Fig. [3. In the full range $0 \leq g_{0}^{2} \leq 1$, they are well 
represented by the expression

$$
z_{T}\left(g_{0}^{2}\right)=\frac{1-0.5090 g_{0}^{2}}{1-0.4789 g_{0}^{2}}
$$

a function which again coincide with the expansion in Eq. (28) to order $g_{0}^{2}$. In this case the error to be attached to the values in Eq. (39) grows linearly from $0.15 \%$ to $0.75 \%$ in the interval $0 \leq g_{0}^{2} \leq 1$. The one loop result agrees with the non-perturbative determination up to $g_{0}^{2} \sim 0.4$ within our statistical errors.

The above results for $Z_{T}\left(g_{0}^{2}\right)$ and $z_{T}\left(g_{0}^{2}\right)$ clearly show that in the range of $g_{0}^{2}$ where the Wilson action is frequently simulated, one-loop perturbation theory is not adequate for computing the renormalization constants of the traceless components of the energy-momentum tensor defined as in Eq. (26). Shifted boundary conditions offer an extremely powerful tool to compute them, and therefore to define non-perturbatively the energy-momentum tensor on the lattice. The strategy implemented here can be easily generalized to QCD, and to (beyond Standard Model) QCD-like or supersymmetric theories.

\section{ACKNOWLEDGMENTS}

We thank H. B. Meyer and D. Robaina for interesting discussions in an early stage of this work. The simulations were performed on the BG/Q at CINECA (INFN and LISA agreement), on the $\mathrm{PC}$ cluster Zefiro of the INFN-CSN4 in Pisa, and on PC clusters at the Physics Department of the University of Milano-Bicocca. We thankfully acknowledge the computer resources and technical support provided by these institutions. This work was partially supported by the INFN SUMA project.

\section{Appendix A: SU(3) conventions}

The Lie algebra of $\mathrm{SU}(3)$ may be identified with the linear space of all hermitian traceless $3 \times 3$ matrices. In the basis $T^{a}, a=1 \ldots 8$, with

$$
\operatorname{Tr}\left[T^{a}\right]=0, \quad T^{a \dagger}=T^{a},
$$

the elements of the algebra are linear combinations of them with real coefficients. The structure constants $f^{a b c}$ in the commutator relation

$$
\left[T^{a}, T^{b}\right]=i f^{a b c} T^{c}
$$

are real and totally anti-symmetric in the indices if the normalization condition

$$
\operatorname{Tr}\left[T^{a} T^{b}\right]=\frac{1}{2} \delta^{a b}
$$

is imposed.

\section{Appendix B: Continuum notation}

In the Euclidean space-time, the path integral of the $\mathrm{SU}(3)$ Yang-Mills theory is defined as

$$
Z=\int D A D \bar{c} D c e^{-S}
$$

where the measures on gauge and ghost fields are defined as usual. The action is defined as

$$
S=\int d^{4} x \mathcal{L}(x), \quad \mathcal{L}=\mathcal{L}^{G}+\mathcal{L}^{G F},
$$

with

$$
\begin{aligned}
\mathcal{L}^{G} & =\frac{1}{2 g_{0}^{2}} \operatorname{Tr}\left[F_{\mu \nu} F_{\mu \nu}\right] \\
\mathcal{L}^{G F} & =\frac{\lambda_{0}}{g_{0}^{2}} \operatorname{Tr}\left[\partial_{\mu} A_{\mu} \partial_{\nu} A_{\nu}\right]+\frac{2}{g_{0}^{2}} \operatorname{Tr}\left[\partial_{\mu} \bar{c} \mathcal{D}_{\mu} c\right]
\end{aligned}
$$

where $g_{0}$ is the bare coupling constant, $\lambda_{0}$ is the gaugefixing parameter, the trace is over the color index and

$$
\begin{aligned}
F_{\mu \nu} & =\partial_{\mu} A_{\nu}-\partial_{\nu} A_{\mu}-i\left[A_{\mu}, A_{\nu}\right], \\
\mathcal{D}_{\mu} c & =\partial_{\mu} c-i\left[A_{\mu}, c\right], \quad A_{\mu}=A_{\mu}^{a} T^{a} .
\end{aligned}
$$

The ghost fields $c$ and $\bar{c}$ are in the adjoint representation of the $\mathrm{SU}(3)$ group, i.e. $c=c^{a} T^{a}$ and analogously for $\bar{c}$.

\section{BRST transformations}

The action (B2) is invariant under the BRST transformations defined as [19 21]

$$
\begin{aligned}
\delta A_{\mu} & =\theta D_{\mu} c \\
\delta \bar{c} & =\lambda_{0} \theta\left(\partial_{\mu} A_{\mu}\right) \\
\delta c & =i \theta c^{2}
\end{aligned}
$$

where $\theta$ is an infinitesimal Grassmann constant. They are nilpotent up to the equations of motion of the ghost field $c$. In fact if we define

$$
\delta \phi=\phi^{\prime}-\phi=\theta \Delta \phi
$$

where $\phi$ is one of the fundamental fields which transforms as in Eqs. (B5), it is easy to prove that 7

$$
\Delta^{2} A_{\mu}=0, \quad \Delta^{2} c=0
$$

while

$$
\Delta^{2} \bar{c}=\lambda_{0} \partial_{\mu} D_{\mu} c
$$

By using Eqs. (B7) and (B8) it is easy to show that the BRST transformations are nilpotent, up to the equation

\footnotetext{
7 To this aim it is useful to notice that $\delta\left(D_{\mu} c\right)=0$.
} 
of motion of $c$, when acting on any product of fundamental fields at arbitrary space-time points and thus on any functional of them.

The gauge-invariant part of the Yang-Mills action (B2) is BRST-invariant because the BRST correspond to infinitesimal gauge transformations with parameter $\theta c(x)$. The gauge-fixing part of the action turns out to be BRSTinvariant too. It can also be written as a BRT rotation of a functional plus a term which, after integrating by parts, is proportional to the equation of motion of $c$ and serves to cancel the term coming from Eq. (B8).

\section{Equations of motion} by

The equations of motion for the gauge field are given

$$
\left\langle\left\{\frac{1}{g_{0}^{2}}\left[\mathcal{D}_{\alpha} F_{\alpha \mu}\right]^{a}+\frac{\lambda_{0}}{g_{0}^{2}} \partial_{\mu} \partial_{\alpha} A_{\alpha}^{a}+\frac{1}{g_{0}^{2}} f^{a b c}\left(\partial_{\mu} \bar{c}^{b}\right) c^{c}\right\}(x) O\right\rangle=-\left\langle\frac{\delta O}{\delta A_{\mu}^{a}(x)}\right\rangle
$$

where $O$ represents a generic string of fields, and the covariant derivative for the adjoint representation is defined as in Eq. (B4), i.e.

$$
\mathcal{D}_{\mu} F_{\mu \nu}=\partial_{\mu} F_{\mu \nu}-i\left[A_{\mu}, F_{\mu \nu}\right]
$$

The equations of motion for the ghosts are

$$
\begin{aligned}
\left\langle\frac{1}{g_{0}^{2}} \partial_{\mu}\left[\mathcal{D}_{\mu} c\right]^{a}(x) O\right\rangle & =-\left\langle\frac{\delta}{\delta \bar{c}^{a}(x)} O\right\rangle \\
\left\langle\frac{1}{g_{0}^{2}}\left[\mathcal{D}_{\mu} \partial_{\mu} \bar{c}\right]^{a}(x) O\right\rangle & =\left\langle\frac{\delta}{\delta c^{a}(x)} O\right\rangle .
\end{aligned}
$$

\section{Appendix C: Energy-momentum tensor in the continuum}

The continuum theory is invariant under the group of space-time translations

$$
x_{\mu}^{\prime}=x_{\mu}-\varepsilon_{\mu}, \quad \phi^{\prime}\left(x_{\mu}^{\prime}\right)=\phi\left(x_{\mu}\right),
$$

where $\phi$ indicates generically one of the fields $A_{\mu}, c, \bar{c}$. The associated WIs can be derived in the usual way by studying the variation of the functional integral under local transformations parameterized by $\varepsilon_{\mu}(x)$

$$
\begin{aligned}
\delta A_{\mu}(x) & =\varepsilon_{\rho}(x) \partial_{\rho} A_{\mu}(x) \\
\delta c(x) & =\varepsilon_{\rho}(x) \partial_{\rho} c(x), \quad \delta \bar{c}(x)=\bar{c}(x) \overleftarrow{\partial}_{\rho} \varepsilon_{\rho}(x)
\end{aligned}
$$

The resulting integrated WIs are

$$
\int d^{4} z \varepsilon_{\nu}(z)\left\langle\partial_{\mu} T_{\mu \nu}^{c}(z) O\right\rangle=-\langle\delta O\rangle
$$

where $\delta O$ is the variation of the string of fields $O$ under the transformation (C2). The canonical energymomentum tensor of the theory can be written as

where

$$
T_{\mu \nu}^{c}=T_{\mu \nu}^{G, c}+T_{\mu \nu}^{G F, c}
$$

$$
\begin{aligned}
T_{\mu \nu}^{G, c} & =\frac{2}{g_{0}^{2}} \operatorname{Tr}\left[F_{\mu \alpha} \partial_{\nu} A_{\alpha}\right]-\delta_{\mu \nu} \mathcal{L}^{G} \\
T_{\mu \nu}^{G F, c} & =\frac{2 \lambda_{0}}{g_{0}^{2}} \operatorname{Tr}\left[\partial_{\alpha} A_{\alpha} \partial_{\nu} A_{\mu}\right] \\
& +\frac{2}{g_{0}^{2}} \operatorname{Tr}\left[\left(\partial_{\mu} \bar{c}\right)\left(\partial_{\nu} c\right)+\left(\partial_{\nu} \bar{c}\right)\left(D_{\mu} c\right)\right]-\delta_{\mu \nu} \mathcal{L}^{F G}
\end{aligned}
$$

For $\varepsilon_{\nu}(z)=\epsilon_{\nu} \delta^{(4)}(z-x)$, Eq. (C3) gives

$$
\epsilon_{\nu}\left\langle\partial_{\mu} T_{\mu \nu}^{c}(x) O\right\rangle=-\left\langle\delta_{x} O\right\rangle,
$$

and when all operators of the string $O$ are localized far away from $x$, the classical conservation identities

$$
\left\langle\partial_{\mu} T_{\mu \nu}^{c}(x) O\right\rangle=0
$$

are recovered. The canonical energy-momentum tensor is neither symmetric nor gauge invariant. To make it both symmetric and gauge invariant one applies the Belinfante procedure, and use the equation of motion. The resulting tensor satisfies the on-shell WIs in Eq. (C8), and it gives the same conserved charges of the canonical tensor when inserted in on-shell correlation functions. The ambiguity left by the use of the equations of motion allows one to define the energy-momentum tensor as the one derived by exploiting the re-parameterization transformations of the theory coupled to an external gravitational field [10, 11, 22]. All definitions related by terms which vanish by the equation of motion are equivalent provided the corresponding contact terms are taken into account in the WIs. The symmetric energy-momentum tensor is defined as

$$
T_{\mu \nu}^{B}=T_{\mu \nu}^{G, B}+T_{\mu \nu}^{G F, B}
$$

where 


$$
\begin{aligned}
T_{\mu \nu}^{G, B}= & \frac{2}{g_{0}^{2}} \operatorname{Tr}\left[F_{\mu \alpha} F_{\nu \alpha}\right]-\delta_{\mu \nu} \mathcal{L}^{G}, \\
T_{\mu \nu}^{G F, B}= & \frac{2 \lambda_{0}}{g_{0}^{2}} \operatorname{Tr}\left[-A_{\mu} \partial_{\nu} \partial_{\alpha} A_{\alpha}-A_{\nu} \partial_{\mu} \partial_{\alpha} A_{\alpha}+\delta_{\mu \nu}\left(\frac{1}{2} \partial_{\alpha} A_{\alpha} \partial_{\beta} A_{\beta}+A_{\alpha} \partial_{\alpha} \partial_{\beta} A_{\beta}\right)\right]+ \\
& \frac{2}{g_{0}^{2}} \operatorname{Tr}\left[\partial_{\mu} \bar{c} D_{\nu} c+\partial_{\nu} \bar{c} D_{\mu} c\right]-\delta_{\mu \nu} \frac{2}{g_{0}^{2}} \operatorname{Tr}\left[\partial_{\alpha} \bar{c} D_{\alpha} c\right] .
\end{aligned}
$$

By comparing Eqs. (C4) and ( $(\mathrm{C} 9)$, it is quite easy to show that

$$
\partial_{\mu} T_{\mu \nu}^{c}=\partial_{\mu} T_{\mu \nu}^{B}+\partial_{\mu}\left\{A_{\nu}^{a}\left[\frac{1}{g_{0}^{2}}\left[D_{\alpha} F_{\alpha \mu}\right]^{a}+\frac{\lambda_{0}}{g_{0}^{2}} \partial_{\mu} \partial_{\alpha} A_{\alpha}^{a}+\frac{1}{g_{0}^{2}} f^{a b c}\left(\partial_{\mu} \bar{c}^{b}\right) c^{c}\right]\right\},
$$

i.e. the two four-divergences differ by terms which are proportional to the equations of motion. If we insert last equation in the WIs (C7) and we use the the equations of motion (B9) we arrive to

$$
\epsilon_{\nu}\left\langle\partial_{\mu} T_{\mu \nu}^{B}(x) O\right\rangle=-\left\langle\delta_{x} O\right\rangle+\epsilon_{\nu} \partial_{\mu}\left\langle A_{\nu}^{a}(x) \frac{\delta O}{\delta A_{\mu}^{a}(x)}\right\rangle .
$$

It is also useful to notice that

$$
T_{\mu \nu}^{G F, B}=\Delta \Xi_{\mu \nu}+\delta_{\mu \nu} \frac{1}{g_{0}^{2}} \operatorname{Tr}\left[\bar{c} \partial_{\alpha} D_{\alpha} c\right]
$$

where $\Delta$ is the BRST variation defined in appendix $B$ and

$$
\begin{aligned}
\Xi_{\mu \nu}= & \frac{2}{g_{0}^{2}} \operatorname{Tr}\left[-A_{\mu} \partial_{\nu} \bar{c}-A_{\nu} \partial_{\mu} \bar{c}+\right. \\
& \left.\delta_{\mu \nu}\left(\frac{1}{2}\left(\partial_{\alpha} A_{\alpha}\right) \bar{c}+A_{\alpha} \partial_{\alpha} \bar{c}\right)\right] .
\end{aligned}
$$

When the interpolating operator $O$ is gauge-invariant, it is thus appropriate to define a gauge-invariant energymomentum tensor

$$
T_{\mu \nu}=T_{\mu \nu}^{G, B}=\frac{1}{g_{0}^{2}}\left\{F_{\mu \alpha}^{a} F_{\nu \alpha}^{a}-\frac{1}{4} \delta_{\mu \nu} F_{\alpha \beta}^{a} F_{\alpha \beta}^{a}\right\}
$$

which satisfies

$$
\left\langle\partial_{\mu} T_{\mu \nu}(x) O\right\rangle=\left\langle\partial_{\mu} T_{\mu \nu}^{B}(x) O\right\rangle
$$

where the term proportional to the equation of motion of the ghosts is null because a gauge-invariant operator is independent of the $\bar{c}$ field. The WIs (C12) applies as well to $T_{\mu \nu}(x)$ without any modification. It is worth nothing that the gauge-invariant energy-momentum tensor generates the very same charges in on-shell correlation functions as all the other definitions in this appendix.

\section{Appendix D: Renormalization of the action density in dimensional regularization}

In this appendix we report the essential formulas in dimensional regularization which are needed in the paper, for a recent review see Ref. [23] and reference therein. In dimensional regularization one replaces $\int d^{4} x \rightarrow \int d^{D} x$, and renormalizes the coupling constant as

$$
g_{0}^{2}=\mu^{2 \epsilon} g^{2} Z_{g}^{-1}
$$

where $D=4-2 \epsilon$. The $\beta$-function is

$$
\begin{aligned}
\tilde{\beta}(\epsilon, g) & =\mu \frac{\partial g}{\partial \mu}=-\epsilon g\left\{1-\frac{g}{2} \frac{\partial}{\partial g} \ln Z_{g}\right\}^{-1} \\
& =-\epsilon g+\beta(g)
\end{aligned}
$$

where

$$
\beta(g)=-g^{3} \sum_{k=0}^{\infty} b_{k} g^{2 k},
$$

and

$$
b_{0}=\frac{1}{(4 \pi)^{2}} \frac{11}{3} N_{c} \quad b_{1}=\frac{1}{(4 \pi)^{4}} \frac{34}{3} N_{c}^{2}
$$

with the number of colours being $N_{c}=3$ in our case.

In presence of shifted boundary conditions it holds

$$
\frac{\partial}{\partial g}\left\langle T_{0 k}\right\rangle_{\boldsymbol{\xi}}=\frac{1}{L_{0}} \frac{\partial g_{0}}{\partial g} \frac{1}{2 g_{0}^{3}} \frac{\partial}{\partial \xi_{k}}\left\langle F_{\alpha \beta}^{a} F_{\alpha \beta}^{a}\right\rangle_{\boldsymbol{\xi}},
$$

which can be written as $\left(x_{0} \neq 0\right)$

$$
\frac{\partial}{\partial g}\left\langle T_{0 k}\right\rangle_{\boldsymbol{\xi}}=\frac{1}{2} \frac{\partial g_{0}}{\partial g} \frac{g^{3}}{g_{0}^{3}} Z_{E}\left[\frac{1}{g^{3}}\left\langle\bar{T}_{0 k}\left(x_{0}\right)\left\{F_{\alpha \beta}^{a} F_{\alpha \beta}^{a}(0)\right\}^{\mathrm{R}}\right\rangle_{\boldsymbol{\xi}, c}\right],
$$


where $Z_{E}$ and the renormalized density are defined in Eq. (16). The expectation values of the renormalized operators in Eq. (D6) are finite and expandable in powers of $g$. By following Ref. [9], see also Ref. [6], the ratio

$$
\mu^{2 \epsilon} \frac{\partial g_{0}}{\partial g} \frac{g^{3}}{g_{0}^{3}} Z_{E}=-\epsilon g \frac{Z_{E} Z_{g}}{\tilde{\beta}(\epsilon, g)}
$$

must then have a series in $g$ with no poles in $\epsilon$. In dimensional regularization the coefficients of the poles in $\epsilon$ in the renormalization constants start at $O\left(g^{2}\right)$, and therefore

$$
\epsilon Z_{E} Z_{g}=\epsilon+R, \quad R=\sum_{k=1}^{\infty} r_{k} g^{2 k},
$$

which implies

$$
-\epsilon g \frac{Z_{E} Z_{g}}{\tilde{\beta}(\epsilon, g)}=\frac{g(\epsilon+R)}{\epsilon g-\beta(g)} .
$$

If we expand in $g$ the denominator we obtain

$$
\frac{g(\epsilon+R)}{\epsilon g-\beta(g)}=\left(1+\frac{R g}{\beta}\right) \sum_{k=0}^{\infty}\left(\frac{\beta}{\epsilon g}\right)^{k}-\frac{R g}{\beta} .
$$

Since this quantity cannot have poles in $\epsilon$

$$
R=-\frac{\beta}{g}
$$

and therefore

$$
\epsilon Z_{E} Z_{g}=\epsilon-\frac{\beta}{g}
$$

The renormalization constant of the energy-density operator is unambiguously fixed from the one of the coupling.

\section{Appendix E: Lattice free theory with shifted boundary conditions}

In this appendix we report the results for the expectation values of $\left\langle T_{0 k}^{[1]}\right\rangle_{\boldsymbol{\xi}},\left\langle T_{00}^{[3]}\right\rangle_{\boldsymbol{\xi}}-\left\langle T_{k k}^{[3]}\right\rangle_{\boldsymbol{\xi}}$ (no sum over k), $\left\langle T_{\mu \mu}^{[2]}\right\rangle_{\xi}$, and $\Delta f / \Delta \xi_{k}$ in the free theory on the lattic 8 . In the infinite volume limit the expectation value of the momentum density is given by [4]

$$
\left\langle T_{0 k}^{[1]}\right\rangle_{\boldsymbol{\xi}}=\frac{8}{L_{0}} \sum_{\ell=0}^{L_{0}-1} \int_{B Z} \frac{d^{3} \boldsymbol{p}}{(2 \pi)^{3}} \frac{\sin \left(p_{0}\right) \sin \left(p_{k}\right) \sum_{\alpha \neq 0, k} \cos ^{2}\left(p_{\alpha} / 2\right)}{4 \sin ^{2}\left(\frac{p_{0}}{2}\right)+\omega_{\boldsymbol{p}}^{2}}
$$

where

$$
\phi_{\boldsymbol{p}}=\boldsymbol{p} \cdot \boldsymbol{\xi}, \quad \omega_{\boldsymbol{p}}^{2}=4 \sum_{k=1}^{3} \sin ^{2}\left(\frac{p_{k}}{2}\right), \quad p_{0}=\frac{2 \pi \ell}{L_{0}}-\phi_{\boldsymbol{p}}
$$

If we notice that [4]

$$
\begin{aligned}
\Sigma\left(\phi, \omega, x_{0}\right) & =\frac{1}{L_{0}} \sum_{\ell=0}^{L_{0}-1} \frac{e^{i x_{0}\left(2 \pi \ell / L_{0}-\phi\right)}}{4 \sin ^{2}\left(\frac{\pi \ell}{L_{0}}-\frac{\phi}{2}\right)+\omega^{2}} \\
& =\frac{1}{2 \sinh \hat{\omega}}\left[\frac{e^{\hat{\omega} x_{0}}}{e^{i L_{0} \phi+L_{0} \hat{\omega}}-1}-\frac{e^{-\hat{\omega} x_{0}}}{e^{i L_{0} \phi-L_{0} \hat{\omega}}-1}\right],
\end{aligned}
$$

where $\omega=2 \sinh (\hat{\omega} / 2)$, and that real and imaginary parts of $\Sigma$ read

$$
\begin{aligned}
& \operatorname{Re} \Sigma\left(\phi, \omega, x_{0}\right)=\frac{\sinh \left(L_{0} \hat{\omega} / 2\right) \cosh \left[\hat{\omega}\left(L_{0} / 2-x_{0}\right)\right]-\sin ^{2}\left(L_{0} \phi / 2\right) \sinh \left(\hat{\omega} x_{0}\right)}{\sinh (\hat{\omega})\left(\cosh \left(L_{0} \hat{\omega}\right)-\cos \left(L_{0} \phi\right)\right)}, \\
& \operatorname{Im} \Sigma\left(\phi, \omega, x_{0}\right)=\frac{-\sin \left(L_{0} \phi\right) \sinh \left(\hat{\omega} x_{0}\right)}{2 \sinh (\hat{\omega})\left(\cosh \left(L_{0} \hat{\omega}\right)-\cos \left(L_{0} \phi\right)\right)},
\end{aligned}
$$

we arrive to

$$
\left\langle T_{0 k}^{[1]}\right\rangle_{\boldsymbol{\xi}}=8 \int_{B Z} \frac{d^{3} \boldsymbol{p}}{(2 \pi)^{3}} \sin \left(p_{k}\right) \operatorname{Im} \Sigma\left(\phi_{\boldsymbol{p}}, \omega_{\boldsymbol{p}}, 1\right) \sum_{\alpha \neq 0, k} \cos ^{2}\left(\frac{p_{\alpha}}{2}\right)
$$

\footnotetext{
8 The lattice spacing is set to $a=1$ in this appendix.
} 
Analogously, for the traceless diagonal component of the energy-momentum tensor we obtain

$$
\begin{gathered}
\left\langle T_{00}^{[3]}\right\rangle_{\boldsymbol{\xi}}-\left\langle T_{k k}^{[3]}\right\rangle_{\boldsymbol{\xi}}=\frac{4}{L_{0}} \sum_{\ell=0}^{L_{0}-1} \int_{B Z} \frac{d^{3} \boldsymbol{p}}{(2 \pi)^{3}} \frac{1}{4 \sin ^{2}\left(\frac{p_{0}}{2}\right)+\omega_{\boldsymbol{p}}^{2}} \times \\
\left\{\left[\cos \left(p_{0}\right)-\cos \left(p_{k}\right)\right] \sum_{\alpha \neq 0, k} \sin ^{2}\left(p_{\alpha}\right)-\left[\cos \left(2 p_{0}\right)-\cos \left(2 p_{k}\right)\right] \sum_{\alpha \neq 0, k} \cos ^{2}\left(\frac{p_{\alpha}}{2}\right)\right\},
\end{gathered}
$$

which by summing over $l$ gives

$$
\begin{aligned}
\left\langle T_{00}^{[3]}\right\rangle_{\boldsymbol{\xi}}-\left\langle T_{k k}^{[3]}\right\rangle_{\boldsymbol{\xi}} & =4 \int_{B Z} \frac{d^{3} \boldsymbol{p}}{(2 \pi)^{3}}\left\{\operatorname{Re} \Sigma\left(\phi_{\boldsymbol{p}}, \omega_{\boldsymbol{p}}, 1\right) \sum_{\alpha \neq 0, k} \sin ^{2}\left(p_{\alpha}\right)-\operatorname{Re} \Sigma\left(\phi_{\boldsymbol{p}}, \omega_{\boldsymbol{p}}, 2\right) \sum_{\alpha \neq 0, k} \cos ^{2}\left(\frac{p_{\alpha}}{2}\right)\right. \\
& \left.+\operatorname{Re} \Sigma\left(\phi_{\boldsymbol{p}}, \omega_{\boldsymbol{p}}, 0\right)\left[\cos \left(2 p_{k}\right) \sum_{\alpha \neq 0, k} \cos ^{2}\left(\frac{p_{\alpha}}{2}\right)-\cos \left(p_{k}\right) \sum_{\alpha \neq 0, k} \sin ^{2}\left(p_{\alpha}\right)\right]\right\} .
\end{aligned}
$$

For the trace part we obtain

$$
\left\langle T_{\mu \mu}^{[2]}\right\rangle_{\boldsymbol{\xi}}=-\frac{16}{L_{0}} \sum_{\ell=0}^{L_{0}-1} \int_{B Z} \frac{d^{3} \boldsymbol{p}}{(2 \pi)^{3}} \frac{\sum_{\alpha, \beta \neq \alpha} \cos ^{2}\left(\frac{p_{\alpha}}{2}\right) \sin ^{2}\left(p_{\beta}\right)}{4 \sin ^{2}\left(\frac{p_{0}}{2}\right)+\omega_{\boldsymbol{p}}^{2}}
$$

which by summing over $l$ gives

$$
\begin{aligned}
\left\langle T_{\mu \mu}^{[2]}\right\rangle_{\boldsymbol{\xi}} & =8 \int_{B Z} \frac{d^{3} \boldsymbol{p}}{(2 \pi)^{3}}\left\{\operatorname{Re} \Sigma\left(\phi_{\boldsymbol{p}}, \omega_{\boldsymbol{p}}, 2\right) \sum_{k=1}^{3} \cos ^{2}\left(\frac{p_{k}}{2}\right)-\operatorname{Re} \Sigma\left(\phi_{\boldsymbol{p}}, \omega_{\boldsymbol{p}}, 1\right) \sum_{k=1}^{3} \sin ^{2}\left(p_{k}\right)\right. \\
& \left.-\operatorname{Re} \Sigma\left(\phi_{\boldsymbol{p}}, \omega_{\boldsymbol{p}}, 0\right) \sum_{k=1}^{3}\left[\sin ^{2}\left(p_{k}\right)+\cos ^{2}\left(\frac{p_{k}}{2}\right)+2 \cos ^{2}\left(\frac{p_{k}}{2}\right) \sum_{q \neq k} \sin ^{2}\left(p_{q}\right)\right]\right\} .
\end{aligned}
$$

In the free theory the discrete derivative of the free energy in Eq. (30) is given by

$$
\frac{\Delta f}{\Delta \xi_{k}}=4 \sum_{\ell=0}^{L_{0}-1} \int_{B Z} \frac{d^{3} \boldsymbol{p}}{(2 \pi)^{3}} \ln \left[\frac{\omega_{\boldsymbol{p}}^{2}+4 \sin ^{2}\left(\left(p_{0}-p_{k} / L_{0}\right) / 2\right)}{\omega_{\boldsymbol{p}}^{2}+4 \sin ^{2}\left(\left(p_{0}+p_{k} / L_{0}\right) / 2\right)}\right]
$$

which by summing over $l$ gives

$$
\frac{\Delta f}{\Delta \xi_{k}}=2 \int_{B Z} \frac{d^{3} \boldsymbol{p}}{(2 \pi)^{3}} \ln \left[\frac{\cosh \left(L_{0} \hat{\omega}_{\boldsymbol{p}}\right)-\cos \left(L_{0} \phi_{\boldsymbol{p}}+p_{k}\right)}{\cosh \left(L_{0} \hat{\omega}_{\boldsymbol{p}}\right)-\cos \left(L_{0} \phi_{\boldsymbol{p}}-p_{k}\right)}\right] .
$$

All previous equations remain valid in finite volume if one makes the substitution

$$
\int_{B Z} \frac{d^{3} \boldsymbol{p}}{(2 \pi)^{3}} \rightarrow \frac{1}{V} \sum_{\boldsymbol{p}}
$$

and defines a prescription for the zero mode.

\section{Appendix F: Numerical results}

For a representative sample of values of $g_{0}^{2}$ that we have simulated we collect the results for the difference of the average plaquettes entering Eq. (33) in Table I] and the values of $\left\langle T_{0 k}^{[1]}\right\rangle_{\boldsymbol{\xi}}$ at $\boldsymbol{\xi}=(1,0,0)$ in Table $\coprod$ The values of $\left\langle T_{0 k}^{[1]}\right\rangle_{\boldsymbol{\xi}}$ and $\left\langle T_{00}^{[3]}\right\rangle_{\boldsymbol{\xi}}-\left\langle T_{k k}^{[3]}\right\rangle_{\boldsymbol{\xi}}$ for $\boldsymbol{\xi}=(1 / 2,0,0)$ are given in Table III.

[1] S. Caracciolo, G. Curci, P. Menotti, and A. Pelissetto, The energy momentum tensor for lattice gauge theories, 


\begin{tabular}{|l|l|l|l|}
\hline$\beta$ & $\frac{1}{18} \sum_{\mu, \nu<\mu}\left[\left\langle\operatorname{ReTr} U_{\mu \nu}\right\rangle_{\boldsymbol{\xi}+a / L_{0} \hat{k}}-\left\langle\operatorname{Re} \operatorname{Tr} U_{\mu \nu}\right\rangle_{\boldsymbol{\xi}-a / L_{0} \hat{k}}\right.$ \\
\hline & $L_{0} / a=3$ & $L_{0} / a=4$ & $L_{0} / a=5$ \\
\hline 6.0 & $5.489(10) \times 10^{-4}$ & $3.028(7) \times 10^{-4}$ & $4.2564(33) \times 10^{-4}$ \\
6.03 & $4.886(10) \times 10^{-4}$ & $2.443(6) \times 10^{-4}$ & $3.8484(38) \times 10^{-4}$ \\
6.125 & $3.601(14) \times 10^{-4}$ & $1.491(7) \times 10^{-4}$ & $1.0016(28) \times 10^{-4}$ \\
6.5 & $1.576(11) \times 10^{-4}$ & $5.160(37) \times 10^{-5}$ & $2.339(20) \times 10^{-5}$ \\
7.0 & $7.65(8) \times 10^{-5}$ & $2.232(33) \times 10^{-5}$ & $8.88(11) \times 10^{-6}$ \\
8.0 & $2.96(5) \times 10^{-5}$ & $7.08(25) \times 10^{-6}$ & $2.43(9) \times 10^{-6}$ \\
9.0 & $1.604(38) \times 10^{-5}$ & $3.60(17) \times 10^{-6}$ & $1.09(11) \times 10^{-6}$ \\
10.0 & $1.041(23) \times 10^{-5}$ & $2.07(8) \times 10^{-6}$ & $6.6(9) \times 10^{-7}$ \\
11.0 & $7.77(22) \times 10^{-6}$ & $1.49(8) \times 10^{-6}$ & $3.8(6) \times 10^{-7}$ \\
12.0 & $5.85(25) \times 10^{-6}$ & $1.06(6) \times 10^{-6}$ & $3.2(5) \times 10^{-7}$ \\
13.5 & $4.29(35) \times 10^{-6}$ & $7.9(8) \times 10^{-7}$ & $2.0(6) \times 10^{-7}$ \\
17.0 & $2.39(14) \times 10^{-6}$ & $3.8(5) \times 10^{-7}$ & $1.3(4) \times 10^{-7}$ \\
20.0 & $1.52(12) \times 10^{-6}$ & $2.8(4) \times 10^{-7}$ & $8.2(28) \times 10^{-8}$ \\
24.0 & $1.14(8) \times 10^{-6}$ & $2.16(27) \times 10^{-7}$ & $4.6(24) \times 10^{-8}$ \\
30.0 & $7.5(6) \times 10^{-7}$ & $1.48(26) \times 10^{-7}$ & $5.5(14) \times 10^{-8}$ \\
50.0 & $2.99(23) \times 10^{-7}$ & $6.6(8) \times 10^{-8}$ & $2.3(7) \times 10^{-8}$ \\
80.0 & $1.25(15) \times 10^{-7}$ & $2.09(39) \times 10^{-8}$ & $0.97(37) \times 10^{-8}$ \\
\hline
\end{tabular}

TABLE I. Values of the difference of the average plaquettes measured at bare coupling $\beta=6 / g_{0}^{2}$ on lattices of size $48^{3} \times$ $L_{0} / a$.

\begin{tabular}{|l|l|l|l|}
\hline$\beta$ & \multicolumn{3}{|c|}{$\left\langle T_{0 k}^{[1]}\right\rangle_{\xi}$} \\
\hline & $\begin{array}{l}L_{0} / a=3 \\
\left(\times 10^{-3}\right)\end{array}$ & $\begin{array}{l}L_{0} / a=4 \\
\left(\times 10^{-3}\right)\end{array}$ & $\begin{array}{l}L_{0} / a=5 \\
\left(\times 10^{-4}\right)\end{array}$ \\
\hline 6.0 & $-5.2735(27)$ & $-1.3772(13)$ & $-2.826(9)$ \\
6.03 & $-5.3921(29)$ & $-1.4447(11)$ & $-4.047(6)$ \\
6.125 & $-5.6976(29)$ & $-1.6064(13)$ & $-5.568(5)$ \\
6.3 & $-6.1359(37)$ & $-1.7977(12)$ & $-6.797(6)$ \\
6.5 & $-6.5124(28)$ & $-1.9495(12)$ & $-7.610(7)$ \\
7.0 & $-7.1554(29)$ & $-2.1899(20)$ & $-8.786(7)$ \\
8.0 & $-7.9077(37)$ & $-2.4488(20)$ & $-9.916(8)$ \\
9.0 & $-8.3673(21)$ & $-2.5947(30)$ & $-10.550(7)$ \\
10.0 & $-8.6896(14)$ & $-2.7002(32)$ & $-10.981(8)$ \\
11.0 & $-8.9385(16)$ & $-2.7780(31)$ & $-11.288(6)$ \\
12.0 & $-9.1331(20)$ & $-2.8358(20)$ & $-11.538(6)$ \\
13.5 & $-9.3654(23)$ & $-2.9111(16)$ & $-11.822(6)$ \\
17.0 & $-9.7261(22)$ & $-3.0181(16)$ & $-12.276(6)$ \\
20.0 & $-9.9253(21)$ & $-3.0862(17)$ & $-12.525(6)$ \\
24.0 & $-10.1097(15)$ & $-3.1420(17)$ & $-12.768(7)$ \\
30.0 & $-10.2941(17)$ & $-3.1987(18)$ & $-12.995(6)$ \\
40.0 & $-10.4792(18)$ & $-3.2587(6)$ & $-13.240(6)$ \\
60.0 & $-10.6608(17)$ & $-3.3148(7)$ & $-13.446(7)$ \\
\hline
\end{tabular}

TABLE II. Values of $\left\langle T_{0 k}^{[1]}\right\rangle_{\boldsymbol{\xi}}$ measured at bare coupling $\beta=$ $6 / g_{0}^{2}$ on lattices of size $48^{3} \times L_{0} / a$ and $\boldsymbol{\xi}=(1,0,0)$.

[2] L. Giusti and H. B. Meyer, Thermal momentum distribution from path integrals with shifted boundary conditions, Phys.Rev.Lett. 106 (2011) 131601, arXiv:1011.2727.

[3] L. Giusti and H. B. Meyer, Thermodynamic potentials from shifted boundary conditions: the scalar-field theory case, JHEP 1111 (2011) 087, arXiv:1110.3136.

[4] L. Giusti and H. B. Meyer, Implications of Poincare symmetry for thermal field theories in finite-volume,

\begin{tabular}{|c|c|c|}
\hline$\beta$ & $\begin{array}{l}\left\langle T_{0 k}^{[1]}\right\rangle_{\boldsymbol{\xi}} \\
\left(\times 10^{-3}\right)\end{array}$ & $\begin{array}{l}\left\langle T_{00}^{[3]}\right\rangle_{\boldsymbol{\xi}}-\left\langle T_{k k}^{[3]}\right\rangle_{\boldsymbol{\xi}} \\
\left(\times 10^{-3}\right)\end{array}$ \\
\hline \multicolumn{3}{|c|}{$L / a=80$} \\
\hline 6.3 & $-4.1926(32)$ & $-5.920(7)$ \\
\hline 7.5 & $-5.2711(17)$ & $-7.228(5)$ \\
\hline 10.0 & $-6.1130(23)$ & $-8.155(6)$ \\
\hline 15.0 & $-6.7612(22)$ & $-8.825(6)$ \\
\hline 24.0 & $-7.2041(9)$ & $-9.2798(23)$ \\
\hline \multicolumn{3}{|c|}{$L / a=120$} \\
\hline 6.3 & $-0.7067(4)$ & $-1.0642(14)$ \\
\hline 7.5 & $-0.9703(5)$ & $-1.4242(14)$ \\
\hline 10.0 & $-1.1345(5)$ & $-1.6321(13)$ \\
\hline 15.0 & $-1.2559(5)$ & $-1.7761(14)$ \\
\hline 24.0 & $-1.3379(5)$ & $-1.8703(14)$ \\
\hline \multicolumn{3}{|c|}{$L / a=160$} \\
\hline 6.3 & $-0.18493(15)$ & $-0.2839(6)$ \\
\hline 7.5 & $-0.29738(16)$ & $-0.4475(5)$ \\
\hline 10.0 & $-0.35095(17)$ & $-0.5190(5)$ \\
\hline 15.0 & $-0.38832(18)$ & $-0.5666(5)$ \\
\hline 24.0 & $-0.41292(22)$ & $-0.5972(6)$ \\
\hline \multicolumn{3}{|c|}{$L / a=240 \quad L_{0} / a=12$} \\
\hline 7.5 & $-0.05643(11)$ & $-0.08597(37)$ \\
\hline 10.0 & $-0.06785(12)$ & $-0.10255(35)$ \\
\hline 15.0 & $-0.07494(16)$ & $-0.1121(5)$ \\
\hline 24.0 & $-0.08029(17)$ & $-0.1188(5)$ \\
\hline
\end{tabular}

TABLE III. Values of $\left\langle T_{0 k}^{[1]}\right\rangle_{\boldsymbol{\xi}}$ and $\left\langle T_{00}^{[3]}\right\rangle_{\boldsymbol{\xi}}-\left\langle T_{k k}^{[3]}\right\rangle_{\boldsymbol{\xi}}$ measured at bare coupling $\beta=6 / g_{0}^{2}$ with shift $\boldsymbol{\xi}=(1 / 2,0,0)$ and at fixed ratio $L / L_{0}=20$.

JHEP 1301 (2013) 140, arXiv:1211.6669.

[5] M. Della Morte and L. Giusti, A novel approach for computing glueball masses and matrix elements in Yang-Mills theories on the lattice, JHEP 1105 (2011) 056, arXiv:1012.2562.

[6] H. Suzuki, Energy-momentum tensor from the Yang-Mills gradient flow, PTEP 2013 (2013), no. 8 083B03, arXiv:1304.0533.

[7] L. Del Debbio, A. Patella, and A. Rago, Space-time symmetries and the Yang-Mills gradient flow, JHEP 1311 (2013) 212, arXiv:1306.1173.

[8] H. Makino and H. Suzuki, Lattice energy-momentum tensor from the Yang-Mills gradient flow-inclusion of fermion fields, PTEP 2014 (2014), no. 6 063B02, arXiv:1403.4772.

[9] M. Lüscher, Small flow-time expansion of gauge-invariant local fields, Unpublished notes June 2013.

[10] S. L. Adler, J. C. Collins, and A. Duncan, Energy-Momentum-Tensor Trace Anomaly in Spin 1/2 Quantum Electrodynamics, Phys.Rev. D15 (1977) 1712.

[11] J. C. Collins, A. Duncan, and S. D. Joglekar, Trace and Dilatation Anomalies in Gauge Theories, Phys.Rev. D16 (1977) 438-449.

[12] S. Caracciolo, P. Menotti, and A. Pelissetto, One loop analytic computation of the energy momentum tensor for lattice gauge theories, Nucl.Phys. B375 (1992) 195-242.

[13] L. Giusti and M. Pepe, Equation of state of a relativistic theory from a moving frame, Phys.Rev.Lett. 113 (2014) 031601, arXiv:1403.0360. 
[14] D. Robaina and H. B. Meyer, Renormalization of the momentum density on the lattice using shifted boundary conditions, PoS LATTICE2013 (2014) 323, arXiv:1310.6075.

[15] N. Cabibbo and E. Marinari, A New Method for Updating $S U(N)$ Matrices in Computer Simulations of Gauge Theories, Phys. Lett. B119 (1982) 387-390.

[16] P. de Forcrand, M. D'Elia, and M. Pepe, A study of the 't Hooft loop in SU(2) Yang-Mills theory, Phys. Rev. Lett. 86 (2001) 1438, hep-lat/0007034.

[17] M. Della Morte and L. Giusti, Exploiting symmetries for exponential error reduction in path integral Monte Carlo, Comput. Phys. Commun. 180 (2009) 813-818.

[18] L. Giusti and M. Pepe, Non-perturbative renormalization of the energy-momentum tensor in
SU(3) Yang-Mills theory, Proc. Sci. LATTICE2014 (2015) 322

[19] C. Becchi, A. Rouet, and R. Stora, The Abelian Higgs-Kibble Model. Unitarity of the S Operator, Phys.Lett. B52 (1974) 344.

[20] C. Becchi, A. Rouet, and R. Stora, Renormalization of the Abelian Higgs-Kibble Model, Commun.Math.Phys. 42 (1975) 127-162.

[21] I. Tyutin, Gauge Invariance in Field Theory and Statistical Physics in Operator Formalism, arXiv:0812.0580

[22] J. Callan, Curtis G., S. R. Coleman, and R. Jackiw, A New improved energy-momentum tensor, Annals Phys. 59 (1970) 42-73.

[23] P. Weisz, Renormalization and lattice artifacts, arXiv: 1004.3462 